\title{
Mesures du bruit produit par les équipements hydrauliques du bâtiment
}

\section{Measurements of noise produced by hydraulic equipement used in the building industry}

\author{
S. Auzou \\ Centre scientifique et technique du bâtiment (CSTB)
}

1. Description du nouveau laboratoire du C.S. T.B.

Le C.S.T.B. s'est doté, à Champs-sur-Marne, d'un nouveau laboratoire de mesure du bruit des équipements hydrauliques, en 1979. Ce laboratoire, en tous points conforme aux spécifications de la norme ISO $3822 / 1$, est composé de deux parties en maçonnerie séparées par une partie en construction légère.

La première partie est le laboratoire proprement dit. Elle comprend : la salle de mesure, d'un volume de $57 \mathrm{~m}^{3}$, montée sur plots élastiques, un sas et la salle d'eau dans laquelle sont installés les équipements à tester (Fig. 1). Il a été posé deux canalisations de mesure, l'une pour le générateur hydraulique de bruit, l'autre pour la robinetterie à l'essai. Cette solution permet d'obtenir une installation très simple tout en maintenant constamment le générateur en eau. Deux manomètres électroniques, un sur chaque canalisation, permettent l'affichage et l'asservissement de la pression. Les mesures s'effectuent en utilisant un microphone placé dans la salle de mesure. Il est également possible de faire les mesures en utilisant un hydrophone. En réalité, puisque l'installation comprend deux canalisations de mesure, il a été disposé un hydrophone miniature dans chacune d'elles (Fig. 2).

La seconde partie est la salle des machines. L'installation fonctionne en circuit fermé et l'élément essentiel est une pompe asservie à l'aide d'un coupleur qui assure sa liaison avec un moteur. La pression utile est comprise entre 0,5 et 8 bars avec un débit maximal de l'ordre de $5 \mathrm{l} / \mathrm{s}$. Trois débitmètres autorisant cinq gammes de débit permettent la lecture de ceux-ci sur un afficheur numérique.

L'intérêt de ce nouveau laboratoire réside dans la très grande souplesse d'utilisation de l'installation hydraulique et l'automatisation aussi poussée que possible du processus d'acquisition des données et d'impression des résultats.
Le laboratoire est destiné aux mesures acoustiques indispensables à l'attribution et au suivi de la marque NF des robinetteries sanitaires ainsi qu'à des travaux de recherche ayant pour but, soit de mieux comprendre les phénomènes en vue de réduire le bruit des équipe-

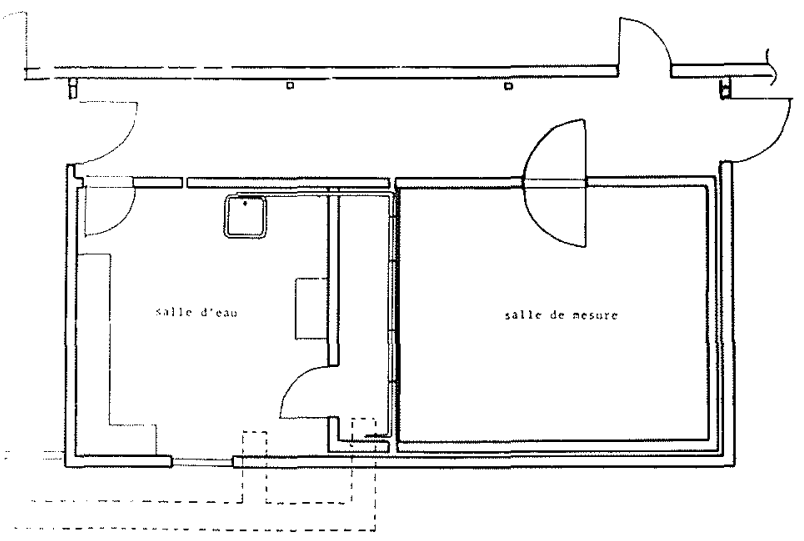

Figure 1

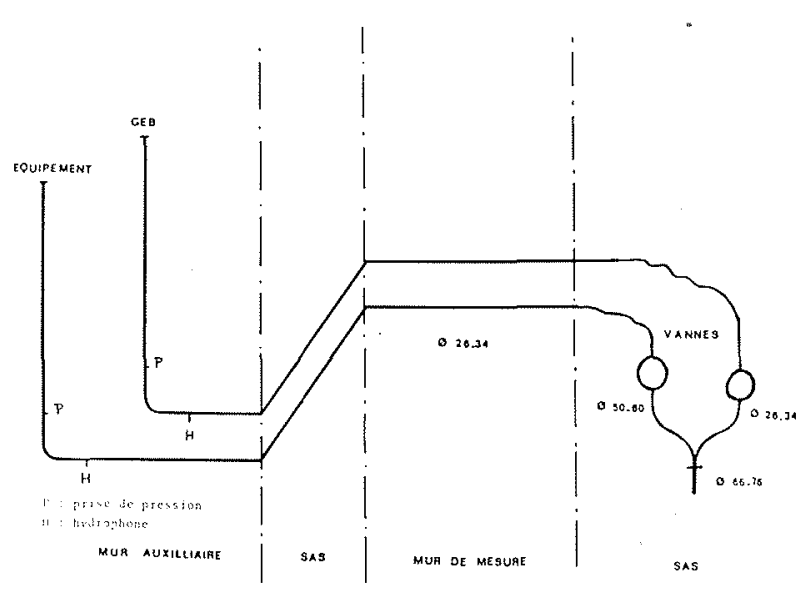

Figure 2. - Vue éclatée des deux canalisations de mesure. 
ments hydrauliques, soit d'imaginer et de tester des codes d'essais d'équipements pour lesquels il n'existe pas encore de procédure d'essai acoustique normalisée.

\section{Principe de la mesure}

Le principe général est le même pour toutes les robinetteries. C'est une mesure par différence, car la valeur obtenue $D_{s}$, qui caractérise une robinetterie, est la différence entre le bruit produit par un générateur hydraulique et la robinetterie testée, sous une pression et pour un débit donnés. En revanche, le générateur est toujours alimenté sous 3 bars.

L'équipement à tester est raccordé à l'extrémité de la canalisation de mesure. Celle-ci est excitée par les turbulences de l'eau et met en vibration une paroi, dite mur de mesure, qui rayonne une pression acoustique dans un local, dit salle de mesure. On mesure de la même manière le bruit produit par un générateur hydraulique de bruit (GEB).

Les robinetteries de puisage sont raccordées à l'extrémité de la canalisation. Pour les mélangeurs et les mitigeurs ceci se fait par l'intermédiaire d'une fourche dont les deux branches ont l'écartement voulu.

Les robinetteries en ligne sont installées à l'extrémité de la canalisation entre deux longueurs de tube du diamètre convenable pour l'équipement testé, d'une longueur égale à dix fois le diamètre. A l'aval, on y raccorde une perte de charge hydraulique silencieuse, constituée d'un long flexible suivi d'une vanne. On entend par silencieux un système dont le bruit propre soit inférieur d'au moins $10 \mathrm{~dB}$ au bruit à mesurer. Cette résistance permet de faire varier le débit de la robinetterie testée.

Pour les robinets de puisage, les clapets de non-retour et les robinets d'arrêt, la pression amont est maintenue constante à 3 bars, puis à 5 bars. Pour les détendeurs, cette pression constante est de 8 bars, la pression aval étant réglée à 3 bars en absence de débit. Cette dernière variera donc plus ou moins en fonction du débit et de la qualité de l'appareil. Dans le cas des détendeurs, on relève la différence de pression $\Delta p$ aux bornes de l'appareil.

Les mesures du niveau de la pression acoustique sont faites à l'aide d'un analyseur temps réel pour chacune des six octaves dont les fréquences centrales s'échelonnent de 125 à $4000 \mathrm{~Hz}$.

On calcule dans un premier temps le niveau de bruit de la robinetterie, $L_{A} P$, valeur obtenue par rapport à une courbe de référence dont le niveau, en $\mathrm{dB}(A)$ est arbitrairement fixé à $45 \mathrm{~dB}(A)$. La différence normalisée $D_{S}$ est donnée par :

$$
\begin{gathered}
D_{S}=45-L_{A P} \\
L_{A F}=10 \lg \sum_{n=1}^{6} 10 \frac{\left(L_{n}+L_{S R n}-\left(L_{S n}-W_{n}\right)+A_{n}\right.}{10}
\end{gathered}
$$

où :

$n$ : indice représentant chacune des six octaves

$L_{n} \quad$ : niveau de la pression acoustique dans l'octave $n$ du bruit produit par la robinetterie

$L_{S R n}:$ valeur dans l'octave $n$ de la courbe de référence
$L_{S n} \quad$ : niveau de la pression acoustique dans l'octave $n$ du bruit produit par le GEB à son emplacement

$A_{n} \quad$ : valeur par octave de la pondération $A$

$W_{n} \quad$ : valeur de la différence par octave entre les niveaux de la pression acoustique dus au GEB à son emplacement et à l'extrémité de la canalisation de mesure destinée à recevoir la robinetterie à tester.

Cette méthode de calcul développée pour la mesure selon ISO 3822/1, c'est-à-dire à partir du niveau de la pression acoustique aérienne, s'applique de la même façon aux mesures faites dans l'eau en utilisant les hydrophones.

\section{Application de la marque NF à la robinetterie}

La marque NF-Robinetterie s'applique actuellement à la robinetterie sanitaire et à la robinetterie de bâtiment depuis plus de dix ans. Elle ne s'appliquait jusqu'en 1974 qu'aux caractéristiques dimensionnelles, générales de construction, physicochimiques, mécaniques et hydrauliques auxquelles doivent satisfaire les robinetteries et ne s'applique encore actuellement qu'aux robinetteries sanitaires et aux aérateurs.

Depuis le $1^{\text {er }}$ janvier 1977 , le classement acoustique accompagne obligatoirement les robinets simples et les mélangeurs.

Toutefois, cette règle ne s'applique pas aux robinets simples de bidets, pour lesquels il n'existe pas encore de méthode d'essai. Depuis le $1^{\text {er }}$ janvier 1978 , ce classement est également obligatoire pour les mitigeurs.

A la fin de 1981, 42 sociétés françaises ou étrangères fabriquant des robinetteries sanitaires étaient titulaires de la marque NF-Robinetterie.

Cette marque forme donc maintenant un tout. Le classement acoustique doit être lisible sur le corps des robinets de même que de l'estampille NF.

Les mesures acoustiques, soit en vue d'une admission, soit pour les contrôles réguliers, auquel cas les robinets sont prélevés chez des dépositaires, portent sur trois exemplaires d'une même robinetterie. L'aérateur, si elle en possède un, est remplacé par une des trois résistances hydrauliques suivantes, caractérisée par leur débit sous 3 bars : $C: 0,50 \mathrm{l} / \mathrm{s}, B: 0,42 \mathrm{l} / \mathrm{s}$ et $A: 0,25 \mathrm{l} / \mathrm{s}$. Deux autres résistances sont actuellement à l'étude, $D: 0,63$ $1 / \mathrm{s}$ et $A A: 0,33 \mathrm{l} / \mathrm{s}$.

- $C$ ou $B$ pour les robinetteries de baignoire;

- $A$ pour les robinetteries de douche (ou les sorties douche ou douchette);

- $B$ ou $A$ pour les autres robinetteries.

La qualité acoustique est caractérisée par la valeur minimale de $D_{s}$, sous la pression de 3 bars. Selon la valeur obtenue le robinet est classé ou non; s'il est classé, il l'est dans l'un des deux groupes suivants :

$$
\begin{aligned}
& \text { Groupe } \mathrm{I}=D_{S} \geqslant 25 \mathrm{~dB}(\mathrm{~A}) \\
& \text { Groupe } \mathrm{II}=25>D_{S} \geqslant 15 \mathrm{~dB}(\mathrm{~A})
\end{aligned}
$$

Les robinets dont le $D_{s}$ est inférieur à $15 \mathrm{~dB}(\mathrm{~A})$ ne sont pas classés, le résultat étant jugé non satisfaisant.

Les robinetteries qui ne sont pas équipées d'un aéra- 
teur sont classées uniquement en fonction du groupe acoustique (I ou II), ou non classées.

Celles qui sont munies d'un aérateur sont classées ainsi :

- d'une part en fonction du groupe acoustique (I ou II), - d'autre part en fonction de la classe de débit (A, B ou C) de la résistance hydraulique avec laquelle elles ont été essayées.

Suivant les cas, une robinetterie peut avoir un seul classement (par exemple IB) ou deux classements (par exemple IIB ou IA). De même, les aérateurs sont marqués avec l'indication de la classe de débit ( $A, B$ ou $C$ ), si la valeur $D_{s}$ de ces aérateurs est supérieure ou égale à $30 \mathrm{~dB}(\mathrm{~A})$. Les aérateurs dont le $D_{s}$ est inférieur à $30 \mathrm{~dB}$ (A) ne seront pas classés, le résultat étant jugé non satisfaisant.

Une robinetterie d'une classe donnée devra être équipée de l'aérateur correspondant. Par exemple, si le corps est marqué IB, elle devra avoir un aérateur de classe $B$. Si cette même robinetterie comporte un aérateur de classe A, elle sera considérée comme classée IA. En aucun cas, elle ne peut être équipée d'aérateur de classe supérieure (par exemple un robinet classé IA ne peut être équipé d'un aérateur de classe B). Par contre, elle peut être équipée d'un aérateur de classe inférieure moyennant un déclassement.

\section{Enseignements tirés de campagnes d'essais}

Ces dernières années ont vu s'améliorer la qualité acoustique des robinets sanitaires de puisage. On peut résumer ainsi la situation, au début du marquage $\mathrm{NF}$ : $35 \%$ des robinetteries sont en classe $1,55 \%$ en classe II et $10 \%$ non classées. L'état actuel peut être résumé en deux nombres : $70 \%$ en classe I et $30 \%$ en classe II, les robinetteries non classées ayant disparu. On peut dire qu'il n'y a plus actuellement de problèmes acoustiques pour les fabricants de robinetteries sanitaires de puisage. Ceci est à mettre à l'actif des fabricants dont un certain nombre se sont dotés soit d'un laboratoire conforme à ISO 3822 , soit d'un montage de mesure simplifié qui permet de dégrossir les problèmes ou de faire du contrôle de fabrication.

Ces équipements sont un excellent moyen de mise au point, permettant ainsi aux fabricants de ne présenter au marquage NF que des robinetteries susceptibles de l'obtenir. Ils peuvent aussi contrôler les phénomènes acoustiques, à défaut de les maitriser.

Compte tenu des résultats et des conclusions obtenus à partir de l'étude faite sur le bruit des robinetteries en ligne, rien ne devrait plus maintenant s'opposer à ce que les normes hydrauliques concernant les détendeurs, les robinets d'arrêt et les clapets de non-retour soient complétées par des critères acoustiques à définir, à partir de mesures effectuées selon le projet de norme ISO $3822 / 3$. Si on adoptait les mêmes critères que ceux retenus pour la robinetterie sanitaire, les deux dernières catégories de robinetteries seraient classées dans le groupe I $\left(D_{s}\right.$ supérieur ou égal à $25 \mathrm{~dB}(\mathrm{~A})$, alors qu'aucun détendeur ne satisferait à la fois à ce critère acoustique et à ceux de la norme hydraulique correspondante (E 29-170).
On remarque que les détendeurs sont des appareils bruyants. Il faut cependant se garder de ne juger leur comportement qu'au vu des résultats acoustiques. En effet, il faut garder à l'esprit la nécessité d'exigences hydrauliques et le jugement, ici comme pour beaucoup d'autres produits, ne peut être que global.

Cette différence du comportement acoustique de ces deux familles d'équipements hydrauliques s'explique, d'une part par la différence des profils internes et d'autre part par la différence de pression aux bornes de la robinetterie, environ 5 bars pour les détendeurs et seulement de l'ordre de 0,2 bar pour les autres robinetteries en ligne.

Il est quelque peu prématuré pour tirer un enseignement sur les résultats acoustiques d'une étude faite sur les robinets thermostatiques de radiateurs, ayant reçu la marque NF, par le C.S.T.B. en 1980.

\section{Comparaison des résultats obtenus par la méthode ISO et par hydrophone}

Il a été proposé et essayé dans quelques pays (France et Allemagne notamment) de mesurer le bruit créé par une robinetterie, non pas selon les dispositions décrites dans la norme ISO $3822 / 1$ et donc dans la norme AFNOR, c'est-à-dire la mesure du niveau de la pression acoustique rayonnée dans une salle, mais de relever le niveau de la pression acoustique directement dans l'eau, à l'aide d'un hydrophone miniature.

Le C.S.T.B. a examiné avec attention les résultats ainsi obtenus et donc les possibilités et les limites de cette méthode alternative. Il a été mené trois expérimentations sur ce sujet. L'une sur des robinets de puisage, qui a permis de faire corréler 1212 couples (Fig. 3), la seconde sur des robinetteries en lignes avec 350 couples (Fig. 4) et enfin la troisième, correspondant à l'étude sur les robinets thermostatiques, a permis l'examen de 1687 couples $D_{S}$ micro/ $D_{S}$ hydro (Fig. 5).

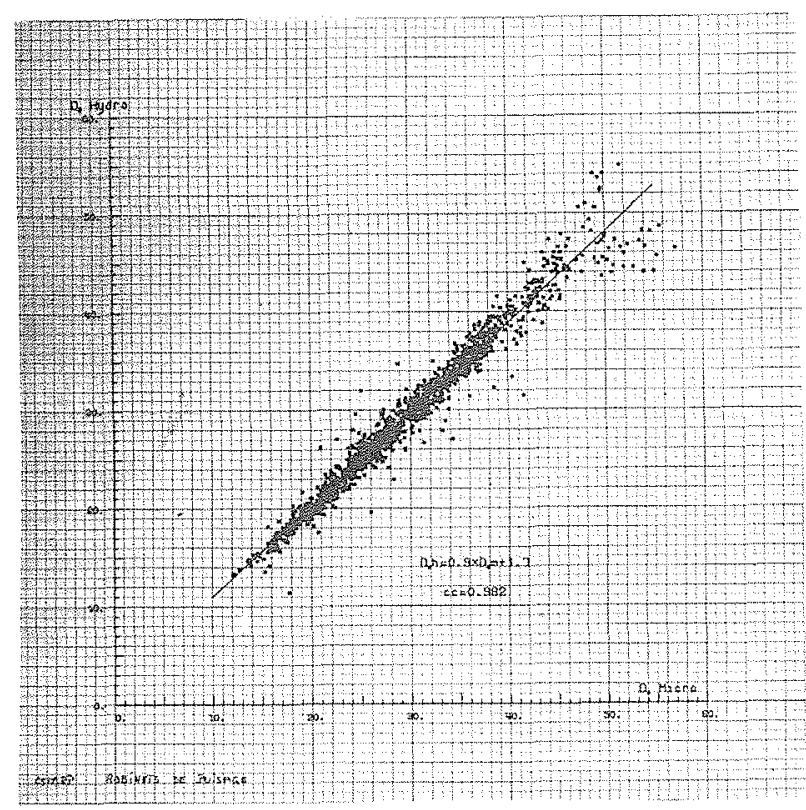

Figure 3. 


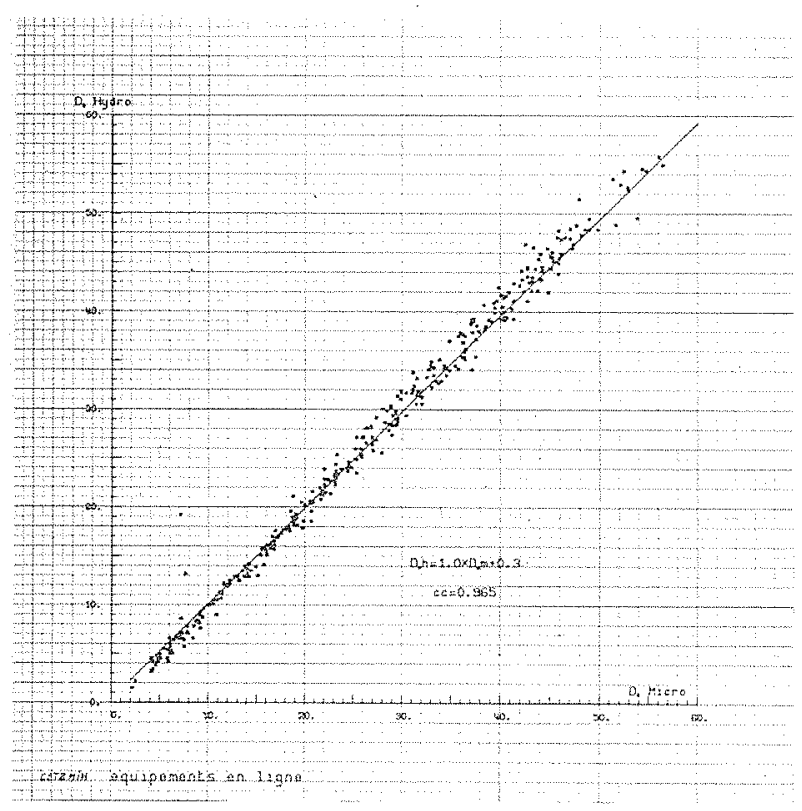

Figure 4

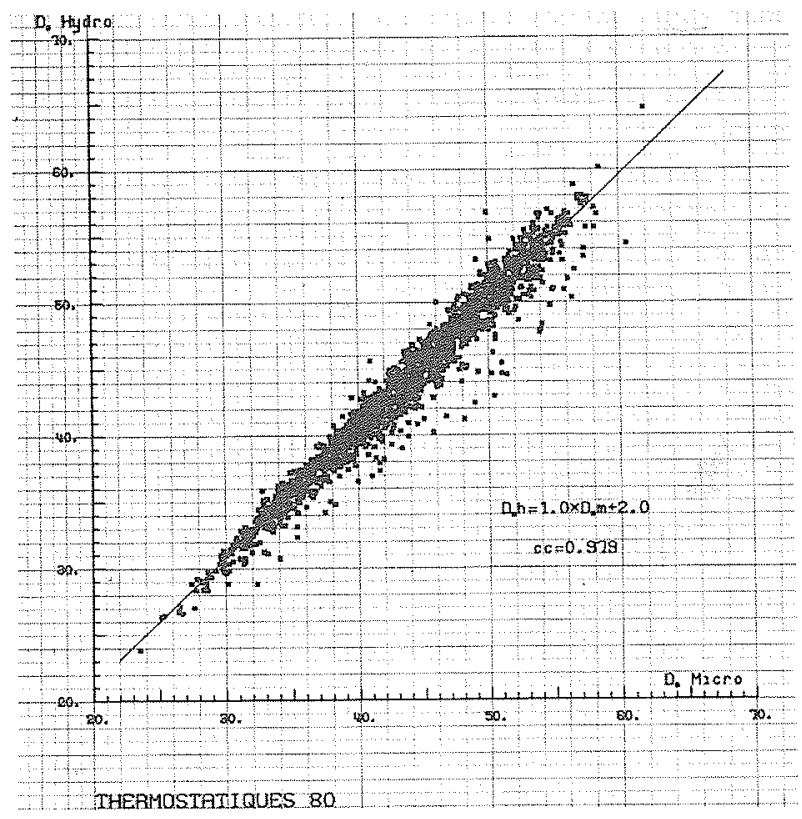

Figure 5
Ces mesures ont été faites en quasi-simultanéité, les deux relevés étant enregistrés immédiatement l'un après l'autre, sans qu'on ait touché à la robinetterie. Dans les deux cas, les mesures sont faites par analyse en octave, avec correction éventuelle due au bruit de fond par octave, et la valeur de $D_{S}$ est obtenue par le calcul, comme expliqué au $\S 2$. On remarque une bonne corrélation d'ensemble, avec un coefficient de corrélation de l'ordre de 0,97. La mesure par hydrophone est cependant optimiste de $2 \mathrm{~dB}$ (A), sauf pour les robinetteries en ligne. On remarque aussi qu'un certain nombre de points s'éloignent de la droite de régression, notamment pour les robinets de puisage et plus particulièrement lorsque les valeurs de $D_{S}$ sont supérieures à $45 \mathrm{~dB}(\mathrm{~A})$.

Dans l'état actuel de nos connaissances, on ne peut pas affirmer que les deux méthodes donnent des résultats identiques. Aussi, tant que les causes de ces écarts ne seront pas élucidées, la méthode par hydrophone, intéressante par une simplification de l'infrastructure et par une moindre sensibilité au bruit de fond, ne peut être utilisée que pour des contrôles et des mises au point, mais pas pour des mesures devant avoir un caractère officiel.

\section{Evolution de la normalisation}

Les travaux de normalisation ont commencé en 1969 , au sein de l'ISO (Organisation Internationale de Normalisation) à partir des projets allerinand et français. Quatre documents étaient prévus à l'intérieur de cette norme, portant le numéro 3822 .
1) La méthode générale.

2) Application aux robinets de puisage.

3) Application aux robinetteries dites en ligne.

4) Application aux autres robinetteries (flotteurs, de chasse, etc. ..).

En 1974, il y a un accord sur la partie 1 et un consensus suffisant sur la partie 2 pour qu'on publie, l'année suivante, deux normes AFNOR correspondantes, sans attendre des documents internationaux définitifs. On a en effet un besoin urgent de ces normes pour pouvoir étendre le domaine de la marque NF des robinets de puisage à leurs caractéristiques acoustiques.

Le projet de norme ISO 3822/1 est révisé, de façon à n'autoriser la mesure directe en $\mathrm{dB}(\mathrm{A})$ qu'à la condition que le spectre du générateur soit parallèle à la courbe de référence, qui légèrement modifiée est incluse dans le corps de la future norme. Cette nouvelle version est adoptée par le sous-comité à Londres en 1978 ainsi que le projet de norme ISO $3822 / 2$. L'accord est obtenu sur le projet ISO $3822 / 3$ à Budapest en 1980. Enfin, les discussions sont en cours au sujet de la partie 4 .

Les normes françaises NF S 31-014, -015 et -016 sont en cours de révision pour correspondre respectivement aux trois parties du document ISO et devraient être rééditées en 1982. En ce qui concerne les robinets à flotteur et les robinets thermostatiques pour radiateurs, on s'achemine, en attendant des normes purement acoustiques, vers l'inclusion d'une annexe dans chacune des deux normes définissant ces produits.

Enfin, on peut penser que le RNE, Réseau National d'Essais, accordera à partir de 1982 des agréments et des habilitations aux laboratoires capables d'effectuer des mesures selon ces trois ou cinq futures normes. 